\title{
Perceptual grouping of objects occupied by target and flankers affects target-flanker interference
}

\author{
Chunming Luo ${ }^{1} \cdot$ Robert W. Proctor ${ }^{2}$
}

Published online: 24 September 2015

(C) The Psychonomic Society, Inc. 2015

\begin{abstract}
We report four experiments that tested whether object-based attentional spreading can be modulated by the perceptual structure of objects occupied by target and flankers in a flanker task. The target and flankers were presented on a single object or three separate objects (of same or different shape), displayed at fixed, known locations. The flanker compatibility effect was larger when the target and flankers were on the same object or on three objects of the same shape than when they were on three objects of different shapes, indicating that perceptual grouping of background objects occupied by target and flankers can affect the target-flanker interference. These results imply that attention likely spreads across different objects occupied by target and flankers when these objects are separated by small gaps or have identical shapes, and attentional spreading is impeded when these objects have different shapes, suggesting that object-based attentional spreading can be modulated by the perceptual structure of objects.
\end{abstract}

Keywords Attention - Object-based attention - Attentional spreading $\cdot$ Search prioritization

A central issue in research on visual attention concerns whether attention is allocated to unparsed regions of space or to perceptual objects (Goldsmith \& Yeari, 2003; Lamy, Leber,

Chunming Luo

luocm@psych.ac.cn

1 Key Laboratory of Behavioral Science, Institute of Psychology, Chinese Academy of Sciences, Beijing, China

2 Department of Psychological Sciences, Purdue University, West Lafayette, IN, USA
\& Egeth, 2013; see Reppa, Schmidt, \& Leek, 2012, for a review). Initial research on visual attention focused on how space constrains the distribution of attention, independently of the structure imposed by objects and surfaces (e.g., Eriksen \& Eriksen, 1974; Posner, 1980; see Chen, 2012, for a review). Subsequently, researchers have concentrated on whether, and how, object perceptual structure (some grouping factors, such as closure, color, contour, and movement rather than space or physical proximity) shapes and guides the allocation of visual attention (Duncan, 1984; Egly, Driver, \& Rafal, 1994; for a review, see Scholl, 2001). Most of those studies have demonstrated that perceptual object structure does indeed modulate visual selection in various contexts, indicating an object-based component of visual attention (e.g., Baldauf \& Desimone, 2014; Hollingworth, Maxcey-Richard, \& Vecera, 2012; Richard, Lee, \& Vecera, 2008).

This object-based selection of attention has been demonstrated in a popular, two-rectangle cueing paradigm developed by Egly et al. (1994). In that study, participants viewed two adjacent vertically or horizontally oriented rectangles - one at either side of the fixation point. Shortly before the target onset, the end of one rectangle was briefly flashed as a cue. On $75 \%$ of the trials (validly cued trials), the target appeared at the cued location (the same location as the cue). On the remaining trials, the target appeared at one of two locations, equally distant from the cued location: (a) the opposite end of the same rectangle (same-object trials) or (b) the nearer end of the other rectangle (different-object trials). Egly et al. (1994) found that target detection was faster in validly cued trials than in invalidly cued ones, implying that location or distance from the cue affected performance - a space-based attention effect. In addition, when invalid-cue trials were examined separately, target detection was faster for same-object targets than for different-object targets, notwithstanding their equivalent distance from the cued location. This result suggests that the 
rectangle also influenced the allocation of attention, indicating an object-based attention effect. Similar results have been observed in other studies (Brown \& Denney, 2007; Lamy \& Egeth, 2002; Lamy \& Tsal, 2000; Luo, Lupiáñez, Funes \& Fu, 2010, 2011; Vecera, 1994).

Object-based attention has also been demonstrated in the flanker paradigm described earlier (Eriksen \& Eriksen, 1974; Eriksen \& Hoffman, 1973). A task-irrelevant distractor letter with the same color as the target letter was allocated more attention than a distractor letter of a different color, even though participants knew the location of the target (Baylis \& Driver, 1992; Harms \& Bundesen, 1983; Kim \& Cave, 2001). Similar results were reported by Kramer \& Jacobson (1991), who found that responses to a central target were affected by task-irrelevant flankers connected to the target, but not by task-irrelevant flankers that did not connect to (i.e., group with) the target.

At least two possible mechanisms for attentional modulation of perceptual efficiency can be used to account for these results. An attentional spreading view of object-based attention proposes that attentional spreading through an attended object can improve the rate and efficiency of perceptual processes, which likely enhances the representation of the attended object relative to unattended objects (Cosman \& Vecera, 2012; Desimone \& Duncan, 1995; Luo et al., 2010, 2011). This view can explain the object-based effects observed in the two-rectangle cueing paradigms (Egly et al., 1994; Lamy \& Tsal, 2000; Vecera, 1994) by proposing that targets displayed in an attended object are more likely to be attended because attention spreads readily within objects but not across objects. Also, this view can explain the objectbased attention effect observed in the flanker tasks reviewed earlier (Baylis \& Driver, 1992; Harms \& Bundesen, 1983; Kim \& Cave, 2001; Kramer \& Jacobson, 1991), by suggesting that attention can spread from the target to the distractors that grouped with the target, whereas spreading would occur little, if at all, from a target to distractors that did not group with the target.

A search prioritization view of object-based attention (e.g., Shomstein \& Behrmann, 2008; Shomstein \& Yantis, 2002, 2004) proposes that there is a predisposition to assign higher priority to searching locations within an already attended object than to searching locations in other objects. Search prioritization is modulated by the extent of target positional certainty (Shomstein \& Yantis, 2002). If a target always appears in the same location, there is no need for attention to search the other locations where the target never appears. Search prioritization can explain many of the findings from the objectbased attention literature. In the two-rectangle cuing task (e.g., Egly et al., 1994; Vecera, 1994), attention would prioritize processing toward the cued location first, followed by the uncued location in the same object, and then toward the uncued location in the other object.
Which of these two object-based attention views, spreading attention or search prioritization, provides a better account for effects of object-based attention? Shomstein \& Yantis (2002) adopted a modified flanker paradigm (Eriksen \& Eriksen, 1974) to address this question. In Experiments 1 to 4 , the target letter was always presented at the center of a central rectangle, with two flanker letters either to the left and right or above and below (see Fig. 1, middle panel). The flanker letters appeared within the same large rectangle in which the target was located or in different small rectangles. Because the target was always located centrally, participants focused their attention locally in the center and did not need search for the target. Shomstein and Yantis reported a flanker compatibility effect that was invariant of whether the target and flankers appeared in the same or different objects. This finding was replicated in Richard et al.'s (2008) Experiments 4 and 5 with a similar paradigm, and the results were taken as evidence of a prioritization account in which the target location was given priority. Because the target letter location was known and fixed, object-based modulation of the flanker effect was not found because search was not required to find the taskrelevant stimuli. By contrast, these results seemed counter to the attentional spreading account, which predicts attentional spreading will occur and enhance target-distractors interference when they appear in the same object but not in different objects.

However, several other studies (e.g., Chen \& Cave, 2006, 2008; Ho, 2011; Richard et al., 2008) have demonstrated that attentional spreading within an object can be observed in the modified flanker paradigm. For example, Richard et al. (2008) adopted a flanker paradigm in their Experiments 1 to 3 similar to that of Shomstein \& Yantis (2002) but used more perceptually integral stimuli (see Fig. 1, left panel). That is, instead of letters superimposed on rectangles, they used rectangles that contained three concavities, in which the central concavity was always the target and the two lateral concavities were the flankers. The target and the flankers could be embedded in a large rectangle (same-object condition) or three small
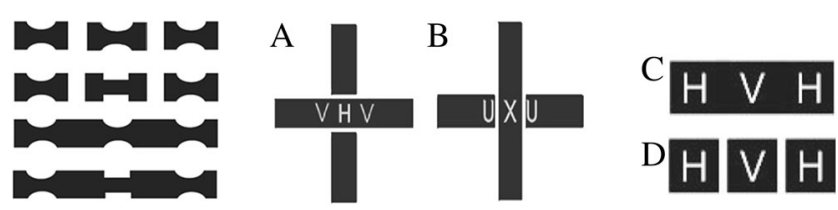

Fig. 1 Left panel: Sample stimuli used in Experiments 1 to 3 of Richard et al. (2008). The top two rows depict stimuli on different-object trials, and the bottom two rows depict stimuli on same-object trials. Within each pair of rows, the target and flanker are compatible in the upper one and incompatible in the lower one. Middle panel: Sample stimuli used by Shomstein \& Yantis (2002). H or V required a left response; V or X required a right response. (A) Same-object compatible response; (B) Different-object compatible response. Right panel: Sample stimuli used in Experiments 4 and 5 of Richard et al. (2008). (C) Same-object compatible response; (D) Different-object compatible response 
rectangles (different-object condition), and they also could have the same shape of concavities (e.g., rectangular; compatible condition) or different shapes (e.g., rectangular target and circular flankers; incompatible condition). A larger flanker compatibility effect was found when the concavities were embedded in the same rectangle rather than different rectangles, which supported the attentional spreading view.

Richard et al. (2008) argued that no modulation of the flanker compatibility effect by object-based attention in Shomstein \& Yantis's (2002, Experiments 1-4) study may have been because the highly familiar letter stimuli allowed for parsing of the targets and flankers away from the background objects, such that the objects were irrelevant to the task. In that case, one would not expect attention spreading to differentiate the results in the two conditions. Richard et al. proposed an "integrality hypothesis" to explain the different effects between their results in Experiments 1 to 3 and those of Shomstein \& Yantis (2002, Experiments 1-4). According to this hypothesis, if taskrelevant features are integral to the objects in a display - that is, when the features contribute to the shape or surface structure of the objects (e.g., Baylis \& Driver, 1992; Behrmann, Zemel, \& Mozer, 1998; Kramer \& Jacobson, 1991) - then objectbased attention may be more highly constrained to select the object via an attentional spread because the features cannot be selected independently of the object. In contrast, if features are not integral to objects - as when a target appears superimposed on the object (e.g., Egly et al., 1994; Shomstein \& Yantis, 2002, 2004; Vecera, 1994) - then object-based attention may be prioritized toward some features over others because the features are readily separated from the objects used to assess objectbased attention.

To summarize, prior studies, using the flanker task found that object-based attention can extend from the target letter to flanking letters that share a basic feature with the target, such as color, closure, motion, good continuation (e.g., Baylis \& Driver, 1992; Driver \& Baylis, 1989; Fuentes, Humphreys, Agis, Carmona \& Catena, 1998; Harms \& Bundesen, 1983; Kim \& Cave, 2001; Kramer \& Jacobson, 1991). Different from those studies, Shomstein \& Yantis (2002) used a modified flanker task in which the target and flankers did not share a basic feature, whereas the target and flankers were displayed in the same large object or three different small objects. Shomstein and Yantis found a flanker compatibility effect invariant of whether the target and flankers appeared in the same or different objects, which seems not to support the attentional spreading account. Richard et al. (2008) adopted a flanker paradigm similar to that of Shomstein \& Yantis (2002) but used more perceptually integral stimuli (see Fig. 1, left panel). Richard et al. found a larger flanker compatibility effect when the target and flankers were embedded in the same rectangles than when they were separately embedded in different rectangles, supporting the attentional spreading account. They proposed an "integrality hypothesis" to explain the different effects between their results in Experiments 1 to 3 and those of Shomstein \& Yantis (2002).

Object-based attention can extend from target letter to flanking letters that share a basic feature, such as closure or color (e.g., Baylis \& Driver, 1992; Driver \& Baylis, 1989; Fuentes et al., 1998; Harms \& Bundesen, 1983; Kim \& Cave, 2001; Kramer \& Jacobson, 1991). Therefore, when the target and flankers are displayed in the same or different objects, perceptual grouping of these background objects occupied by target and flankers may affect the target-flankers interference. We refer to this possibility as the perceptual grouping account. This account may explain why no modulation of flanker effect by objects occurred in Shomstein \& Yantis's (2002) Experiments 1 to 4 and Richard et al.'s (2008) Experiments 4 and 5: Because the objects used in those experiments were similar or identical in the different-object condition, a large extent of perceptual grouping and attention could extend across them.

In four experiments, we distinguished the search prioritization, integrality, and perceptual grouping accounts. In Experiments 1 to 3, we investigated whether perceptual grouping of background objects occupied by target and flankers can affect target-flanker interference. Moreover, in those experiments we used an arrow-flanker task in which the target and two flankers were arrows with identical or inverse directions, and the arrows were presented on the same object or different objects. Responses were made according to the direction of the central arrow while attempting to ignore the irrelevant distracting arrows on both sides (i.e., flankers). The direction of the flanking arrows could be congruent $(\leftarrow \leftarrow \leftarrow)$ or incongruent $(\longleftarrow \longleftrightarrow \leftarrow)$ with respect to the central arrow. In the incongruent trials, there typically is a cost in (RTs) because of the difficulty in ignoring the conflicting flankers (Weinbach \& Henik, 2012). The manipulation was helpful to examine the integrality hypothesis, that is, whether, when the target and flankers are not integral to the objects in a display, the effect of object-based attention is not observed. In Experiments 4A and 4B, we used the same-object and different-object stimuli from Experiments 2 and 3, respectively, and the letter-identity flanker task of Richard et al.'s Experiments 4 and 5 and Shomstein \& Yantis (2002), in order to verify that the pattern of results in Experiments 2 and 3 could be obtained when the stimuli were not arrows. The obtained results in the present study help to understand the mechanisms that are responsible for object-based attentional selection.

\section{Experiment 1}

In this experiment, we used a display as shown in Fig. 2. A large object was used in the same-object condition, whereas in 


\section{Compatible Incompatible}

Same Object

Experiment 1.

Different Object
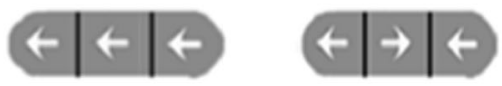

Same Object
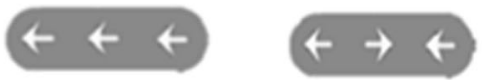

Experiment 2.

Different Object
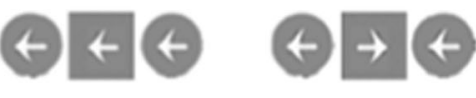

Same Object

Experiment 3.

Different Object

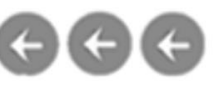

(4) $\rightarrow$

Same Object

Experiment $4 \mathrm{~A}$

Different Object

Same Object
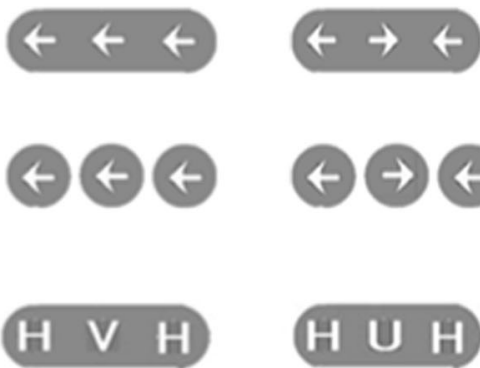

$\oplus \nabla \oplus \Theta \nabla \Theta$

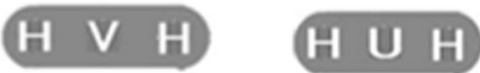

Experiment 4B

\section{Different Object H $\mathrm{H}$ H $\mathrm{H} H$}

Fig. 2 Sample same- and different-object stimuli, with compatible and incompatible flankers, used in Experiments 1 to 4 for the horizontal display

the different-object condition the large object was divided into three small objects by two gaps. Three arrows superimposed on these objects were used as target and flankers. Therefore, the task-relevant features were not integral to the objects in this display. The main aim was to test the perceptual grouping account. According to this account, the three small objects occupied by target and flankers separated by two small gaps can allow attention to extend to groups of objects. Consequently, the flanker compatibility effect would be no different in the same- and different-object conditions. Another objective was to investigate whether we could replicate the findings of Shomstein \& Yantis's (2002) study for which there was no modulation of flanker compatibility effect by whether the target and flankers appeared in the same object or in different objects.
The search prioritization view predicts that the flanker compatibility effect should not vary across the same-object and different-object conditions, because the target location was fixed. The integrality hypothesis predicts that the flanker compatibility effect should be no different in the same- and different-object conditions, because the targets and flankers are not parts of the objects, and attention spreading cannot influence the flanker compatibility.

\section{Method}

Participants Twenty-six undergraduate students (14 males, 12 females) took part in the experiment for payment. All participants in this and the other experiments were from universities near the Institute of Psychology, Chinese Academy of 
Sciences, and they had normal or corrected-to-normal vision and were naïve as to the purpose of the experiment.

Apparatus and stimuli Stimuli were presented on a super VGA high-resolution color monitor with a black background. A computer, running E-Prime 1.1 software, controlled the presentation of stimuli, timing operations, and data collection. Participants viewed the monitor from a distance of approximately $57 \mathrm{~cm}$ in a dimly lit room.

The fixation point was a central $0.3^{\circ} \times 0.3^{\circ}$ white asterisk. As shown in Fig. 2, the horizontal main display consisted of a large object that subtended $4.2^{\circ} \times 1.3^{\circ}$ for the same-object trials and three small objects (each subtended $1.3^{\circ} \times 1.3^{\circ}$ ) for the different-object trials. The large object was constructed from a rectangle $\left(2.9^{\circ} \times 1.3^{\circ}\right)$ and two half circles (each subtended $0.65^{\circ} \times 1.3^{\circ}$ ). For the different-object condition, the middle small object was a square $\left(1.3^{\circ} \times 1.3^{\circ}\right)$ and the other two objects were constructed from a half circle and a rectangle (each subtended $0.65^{\circ} \times 1.3^{\circ}$ ), and the separation of two adjacent objects was $0.15^{\circ}$. Three arrows (each with $0.7^{\circ}$ $\times 0.7^{\circ}$ of visual angle) appeared on the large object for the same-object trials or appeared separately on the three small objects for the different-object trials (see Fig. 2). The target arrows always appeared at the center of the display, and the flanker arrows were $0.95^{\circ}$ far from the center of screen. The main displays in which the target and flankers were arrayed horizontally are shown in Fig. 2, and the main displays in which they were arrayed vertically were obtained by rotating the horizontal displays $90^{\circ}$ counterclockwise.

Procedure and design Each trial began with a white fixation asterisk for $1 \mathrm{~s}$, replaced by the main display, which remained visible until the participant responded or for $1 \mathrm{~s}$ if there was no response. This terminated the current trial, and the next one began after a 1-s intertrial interval during which the screen was black.

There were two trial blocks, one for the horizontal displays and the other for the vertical displays, with a rest interval of $30 \mathrm{~s}$ between the two blocks. Each block consisted of 16 practice trials followed by 128 test trials. In the trial block for the horizontal display, the task was to press the $\mathrm{C}$ key on the bottom row of the computer's keyboard when the middle arrow pointed to the left and the M key when it pointed to the right; in the trial block for the vertical display, the task was to press the $\mathrm{C}$ key when the middle arrow pointed down and the $\mathrm{M}$ key when it pointed up (this mapping was used because it tends to be more compatible than the opposite mapping; Cho $\&$ Proctor, 2003). The order of the two trial blocks was counterbalanced across participants. The response keys and computer screen were aligned such that the fixation asterisk and the midway point between the two response keys were on the participant's sagittal midline. Participants were firmly instructed to maintain fixation and to respond as quickly and accurately as possible.

\section{Results}

Mean RTs for the correct trials and PEs were calculated for each participant as a function of display orientation (horizontal, vertical), object condition (same, different), and flanker compatibility (compatible, incompatible; see Tables 1 and 2). They then were submitted to separate analyses of variance (ANOVAs).

Reaction time Responses were faster for the horizontal display $(426 \mathrm{~ms})$ than for the vertical display $(481 \mathrm{~ms}), F(1,25)=$ 32.36, $p<.001, M S E=4,811, \eta_{p}{ }^{2}=.564$, in the differentobject condition $(450 \mathrm{~ms})$ than in the same-object condition (457 ms), $F(1,25)=9.56, p=.005, M S E=263, \eta_{p}{ }^{2}=.277$, and in the compatible condition (441 ms) than in the incompatible condition (465 ms), $F(1,25)=43.41, p<.001, M S E=$ $681, \eta_{p}{ }^{2}=.635$. The interaction between object condition and flanker compatibility was not significant, $F<1$. Further analysis showed that the flanker compatibility effects were reliable in the same-object $(26 \mathrm{~ms})$ and different-object $(22 \mathrm{~ms})$ conditions, $t(25)=7.06, p<.001 ; t(25)=4.40, p<.001$. The other interactions were not significant $\left(F_{\mathbf{S}}<1\right.$; see Fig. 3 ).

Table 1 Experiments 1 to 4: Mean Reaction Time (in ms) and Standard Deviation (in parentheses) as a Function of Display Orientation, Object Condition, and Flanker Compatibility Note. $\mathrm{SO}=$ same object; $\mathrm{DO}=$ different object

\begin{tabular}{|c|c|c|c|c|c|}
\hline & & \multicolumn{2}{|l|}{ Horizontal } & \multicolumn{2}{|l|}{ Vertical } \\
\hline & & SO & DO & $\mathrm{SO}$ & DO \\
\hline \multirow[t]{3}{*}{ Exp. 1} & Compatible & $414(75)$ & $413(75)$ & 474 (104) & 465 (100) \\
\hline & Incompatible & 443 (77) & 434 (76) & $496(112)$ & 487 (104) \\
\hline & Effect size & $29 * *$ & $21 * *$ & $22 * *$ & $22^{*}$ \\
\hline \multirow[t]{3}{*}{ Exp. 2} & Compatible & $427(78)$ & $425(86)$ & $451(101)$ & $456(108)$ \\
\hline & Incompatible & $456(93)$ & $442(90)$ & $464(105)$ & 452 (107) \\
\hline & Effect size & $29 * *$ & $17 * *$ & $13^{*}$ & -4 \\
\hline \multirow[t]{3}{*}{ Exp. 3} & Compatible & 457(106) & $452(100)$ & $480(117)$ & $470(109)$ \\
\hline & Incompatible & 484(111) & 477 (112) & 498(117) & $496(127)$ \\
\hline & Effect size & $27^{* *}$ & $25^{* *}$ & $18^{* *}$ & $26^{* *}$ \\
\hline \multirow[t]{3}{*}{ Exp. 4A } & Compatible & $551(107)$ & $546(112)$ & & \\
\hline & Incompatible & $573(118)$ & $553(118)$ & & \\
\hline & Effect size & $22 * *$ & 7 & & \\
\hline \multirow[t]{3}{*}{ Exp. 4B } & Compatible & $554(143)$ & $549(152)$ & & \\
\hline & Incompatible & $578(166)$ & $568(155)$ & & \\
\hline & Effect size & $24 * *$ & $19^{* *}$ & & \\
\hline
\end{tabular}

$* p<.05 .{ }^{*} p<<.001$. 
Table 2 Experiments 1 to 4: Percent error (\%) and Standard Deviation (in parentheses) as a Function of Display Orientation, Object Condition, and Flanker Compatibility Note. $\mathrm{SO}=$ same object; $\mathrm{DO}=$ different object

\begin{tabular}{|c|c|c|c|c|c|}
\hline & & \multicolumn{2}{|c|}{ Horizontal } & \multicolumn{2}{|l|}{ Vertical } \\
\hline & & SO & DO & SO & DO \\
\hline \multirow[t]{3}{*}{ Exp. 1} & Compatible & $0.1(0.05)$ & $0.2(0.05)$ & $2.8(0.16)$ & $4.6(0.20)$ \\
\hline & Incompatible & $0.7(0.08)$ & $0.6(0.08)$ & $6.5(0.24)$ & $6.6(0.24)$ \\
\hline & Effect size & 0.60 & 0.40 & 3.70 & 2.00 \\
\hline \multirow[t]{3}{*}{ Exp. 2} & Compatible & $2.0(0.14)$ & $0.8(0.09)$ & $2.6(0.16)$ & $2.4(0.15)$ \\
\hline & Incompatible & $1.9(0.14)$ & $2.0(0.14)$ & $5.2(0.21)$ & $4.9(0.21)$ \\
\hline & Effect size & -0.10 & 1.20 & 2.60 & 2.50 \\
\hline \multirow[t]{3}{*}{ Exp. 3} & Compatible & $2.0(0.14)$ & $2.5(0.16)$ & $2.8(0.16)$ & $2.4(0.15)$ \\
\hline & Incompatible & $4.6(0.21)$ & $4.4(0.21)$ & $2.4(0.15)$ & $4.1(0.20)$ \\
\hline & Effect size & 2.60 & 1.90 & -0.40 & 2.70 \\
\hline \multirow[t]{3}{*}{ Exp. 4A } & Compatible & $4.7(0.22)$ & $3.8(0.19)$ & & \\
\hline & Incompatible & $4.9(0.22)$ & $4.4(0.20)$ & & \\
\hline & Effect size & 0.20 & 0.60 & & \\
\hline \multirow[t]{3}{*}{ Exp. 4B } & Compatible & $3.1(0.17)$ & $4.4(0.18)$ & & \\
\hline & Incompatible & $4.0(0.20)$ & $3.8(0.19)$ & & \\
\hline & Effect size & 0.90 & -0.60 & & \\
\hline
\end{tabular}

${ }^{*} p<.05 .{ }^{* *} p<.001$

Percent error The main effects of object condition and flanker compatibility were not significant, $F(1,25)=1.23, p=.278$, $M S E=.001, \eta_{p}{ }^{2}=.047, F(1,25)=1.77, p=.196, M S E=$ $.008, \eta_{p}{ }^{2}=.066$, but there was a main effect of display orientation, $F(1,25)=6.45, p=.018, M S E=.018, \eta_{p}{ }^{2}=.205$. PE was smaller with the horizontal display $(0.4 \%)$ than with the vertical display $(5.1 \%)$. The interaction between object condition and display orientation was not significant, $F(1,25)=1.46, p=.238$, $M S E=.001, \eta_{p}{ }^{2}=.055$, and none of the other interactions was reliable $(F \mathrm{~S}<1)$.

\section{Discussion}

In this experiment, the three small objects were identical in part and the task-relevant features were not integral to the objects and did not contribute to the shape or surface structure of the objects. We observed that participants responded faster when targets and flankers were compatible than when they were incompatible, indicating a flanker compatibility effect. Responses were slower when all the arrows appeared within the same object than when they appeared in different objects, showing an opposite object-based attention effect from that in previous studies (e.g., Chen \& Cave, 2006, 2008; Shomstein $\&$ Yantis, 2002). This result likely reflects a segmentation process that is required to separate irrelevant information (i.e., the flankers) from the relevant information (i.e., the target) when the items are within the same object; this segmentation stage may not be required when the flankers appear in different objects, because they are already perceptually segmented from the object occupied by the target (e.g., Chen \& Cave, 2006, 2008; Shomstein \& Yantis, 2002, 2004).

As observed in Shomstein \& Yantis (2002), the flanker compatibility effect did not vary with whether the target and flankers appeared on the same object, and it was significant in the two conditions. This result is consistent with the perceptual grouping account, given that the three small objects are similar and attention may spread from the object occupied by the target to the objects occupied by the flankers, which could result in the modulation of attentional spreading on the flanker compatibility effect not being observed.

This result seems to support the search prioritization view, given that the target location is fixed. Also, the result could be explained by the integrality hypothesis, as the task-relevant features are not integral to the objects in a display, likely eliminating the effect of objects on target-distractor interference.

\section{Experiment 2}

In this experiment, we used displays similar to those in Experiment 1, except that for the different-object condition the three small objects were two identical circles and a square (see Fig. 2). Therefore, the displays used in this experiment were different from Experiment 1 and those in Shomstein \& Yantis's (2002) study (see Fig. 1, right panel) in that the three small objects were not identical in this experiment.

The predictions by the search prioritization view and integrality hypothesis were identical to those in Experiment 1, respectively. However, the perceptual grouping account predicts that the flanker compatibility effect would be larger in the same-object condition than in the different-object condition, as the three small objects being different in shape, would impede attention from extending from the object occupied by the target to the objects occupied by flankers.

\section{Method}

Participants Twenty-six undergraduate students (10 males, 16 females) took part in the experiment for payment.

Apparatus, stimuli, procedure and design The apparatus, stimuli, procedure, and design were identical with Experiment 1, with the exception that for the different-object condition, the left and right objects were replaced by two circles (each subtended $1.3^{\circ} \times 1.3^{\circ}$ ). 


\section{Results}

Mean RTs for the correct trials and PEs were calculated for each participant as a function of display orientation (horizontal, vertical), object condition (same, different), flanker compatibility (compatible, incompatible; see Tables 1 and 2). Then, each measure was submitted to a separate ANOVA.

Reaction time Responses were faster for the horizontal display $(437 \mathrm{~ms})$ than for the vertical display $(456 \mathrm{~ms}), F(1,25)=$ $8.71, p=.007, M S E=2,055, \eta_{p}{ }^{2}=.258$, in the different-object condition (444 ms) than in the same-object condition (450 $\mathrm{ms}), F(1,25)=6.00, p=.022, M S E=303, \eta_{p}{ }^{2}=.195$, and when the flankers were compatible with the target $(440 \mathrm{~ms})$ than when they were incompatible (454 ms), $F(1,25)=$

\section{Experiment 1}

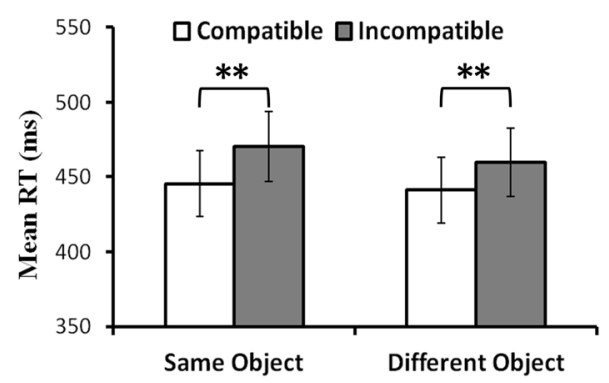

\section{Experiment 3}

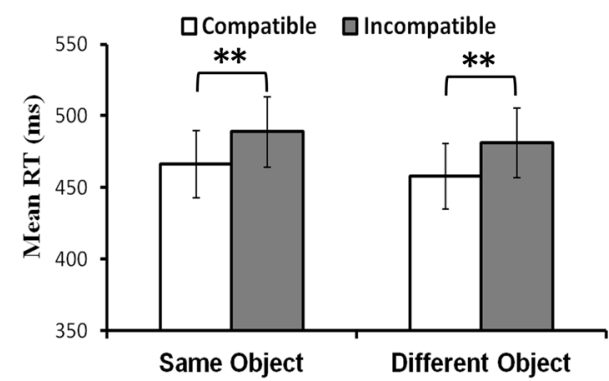

Experiment 4B

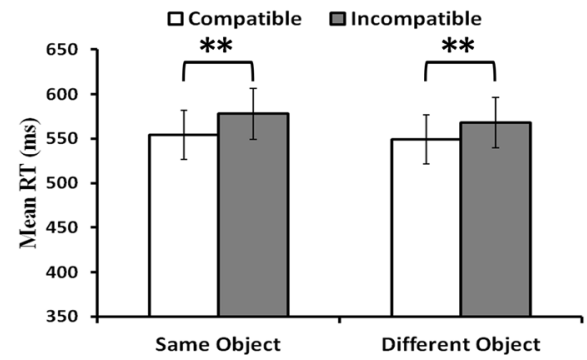

Fig. 3 Mean reaction time (RT) as a function of object condition and flanker compatibility in Experiments 1 to 4 . The data in Experiments 1 to 3 are collapsed across vertical and horizontal orientations of the display.
13.51, $p=.001, M S E=746, \eta_{p}{ }^{2}=.351$. Unlike Experiment 1 , object condition interacted with flanker compatibility, $F(1$, $25)=12.36, p=.002, M S E=213, \eta_{p}^{2}=.331$, with the flanker compatibility effect being larger in the same-object condition (21ms) than in the different-object condition (7 ms; see Fig. 3), $t(25)=3.52, p=.002$. Further analysis showed that the flanker compatibility effect was significant in the same-object condition, $t(25)=4.43, p<.001$, but not in the different-object condition, $t(25)=1.80, p=.085$. Also, display orientation interacted with flanker compatibility, $F(1,25)=18.45, p<$ $.001, M S E=224, \eta_{p}^{2}=.425$, with the flanker compatibility effect evident for the horizontal display $(23 \mathrm{~ms})$ but not the vertical display $(5 \mathrm{~ms})$. The interaction between display type and object condition was not significant, $F(1,25)=1.96, p=$ $.174, M S E=130, \eta_{p}^{2}=.073$, nor was the three-way interaction of all variables, $F<1$.

\section{Experiment 2}

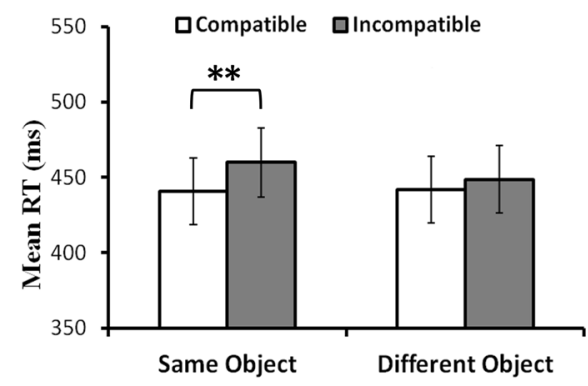

\section{Experiment 4A}

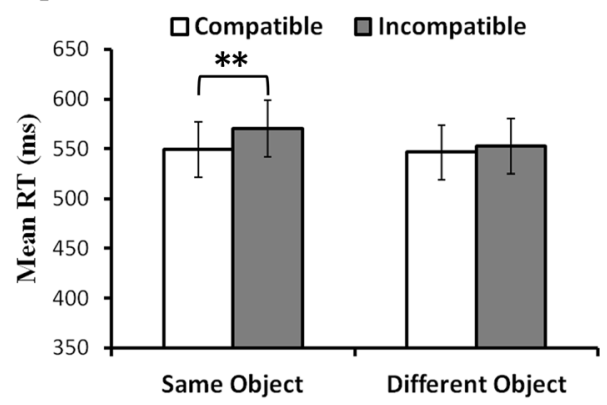

In all of the graphs the error bars are within-subject $95 \%$ confidence intervals on the same-object versus different-objects comparisons (Cousineau, 2007) 
Although the three-way interaction was not significant, to ensure a reliable interaction between object condition and flanker compatibility for each display orientation, we still performed separate analyses for each display orientation with object condition and flanker compatibility as within-subject variables. The analyses showed that the flanker compatibility effect was larger in the same-object condition than in the different-object condition for the horizontal display $(29 \mathrm{~ms}$ vs. $17 \mathrm{~ms}), t(25)=2.28, p=.035$, and the vertical display $(13 \mathrm{~ms}$ vs. $-4 \mathrm{~ms}), t(25)=3.05, p=.005$.

Percent error The main effect of object condition was not significant, $F(1,25)=1.31, p=.264, M S E=.001, \eta_{p}{ }^{2}=.050$, but the main effect of display orientation was, $F(1,25)=5.65$, $p=.025, M S E=.004, \eta_{p}{ }^{2}=.184$, and that of flanker compatibility approached significance, $F(1,25)=4.17, p=.052$, $M S E=.003, \eta_{p}{ }^{2}=.143$. PE was smaller for the horizontal display $(1.7 \%)$ than the vertical display $(3.8 \%)$, and tended to be lower in the compatible condition $(2.0 \%)$ than in the incompatible condition (3.5\%). All interactions were not reliable $(p s>.253)$.

\section{Discussion}

The results of this experiment replicate those of earlier studies showing that participants respond faster when the target and flankers are compatible than when they are incompatible. Responses were slower when all the arrows appeared within the same object than when they appeared in different objects, replicating the results observed in Experiment 1 and other studies (e.g., Chen \& Cave, 2006; Richard et al., 2008, Experiments 4 and 5; Shomstein \& Yantis, 2002; Zhao, Kong, \& Wang, 2013).

The flanker compatibility effect was greater when the arrows appeared within the same object than when they appeared in different objects, which is consistent with the prediction of the perceptual grouping account. The larger flanker compatibility effect in the same-object condition may be due to the three small objects being different, which impedes object-based attention from extending from the object occupied by the target to the objects occupied by the flankers. This result does not fit with the search prioritization view and integrality hypothesis.

\section{Experiment 3}

In Experiment 3 we used the same display as in Experiment 2 except that, for the different-object condition, we changed the central small object to a circle, rendering all three small objects identical circles. This display was similar to that used in Experiments 4 and 5 of Richard et al. (2008; see Fig. 1), in which the small objects were identical squares.

The predictions of the search prioritization view and integrality hypothesis are identical to those in Experiments 1 and 2. The perceptual grouping account predicts that the flanker compatibility effect would be no different in the same- and different-object conditions because the three small objects were identical, which may allow object-based attention be able to extend to the group of objects.

\section{Method}

Participants Twenty-six undergraduate students (12 males, 14 females) took part in the experiment for payment.

Apparatus, stimuli, procedure, and design The apparatus, stimuli, procedure, and design are identical with Experiment 3 , except that for the different-object condition, the middle square was replaced by a circle with $1.3^{\circ} \times 1.3^{\circ}$ of visual angle.

\section{Results}

Mean RTs for the correct trials and PEs were calculated for each participant as a function of display orientation (horizontal, vertical), object condition (same, different), flanker compatibility (compatible, incompatible; see Tables 1 and 2). They were then submitted to separate ANOVAs.

Reaction time The RT data yielded significant main effects of display, $F(1,25)=10.72, p=.003, M S E=1,700, \eta_{p}{ }^{2}=.300$, object condition, $F(1,25)=6.60, p=.017, M S E=262, \eta_{p}{ }^{2}=$ .209 , and flanker compatibility, $F(1,25)=30.49, p<.001$, $M S E=990, \eta_{p}{ }^{2}=.550$. As shown in Fig. 3, the interaction between object condition and flanker compatibility was not significant, $F<1$. Further analysis showed that the flanker compatibility effects were reliable in the same-object ( 23 $\mathrm{ms})$ and different-object $(25 \mathrm{~ms})$ conditions, $t(25)=6.18, p$ $<.001 ; t(25)=4.28, p<.001$. The other two-way interactions $(F \mathrm{~S}<1)$ and the only three-way interaction, $F(1,25)=1.58, p$ $=.220, M S E=188, \eta_{p}{ }^{2}=.059$, were not significant.

Percent error The main effect of flanker compatibility was not significant, $F(1,25)=2.87, p=.103, M S E=.004, \eta_{p}{ }^{2}=$ .103 , nor were the other main effects $\left(F_{\mathrm{S}}<1\right)$. The three-way interaction was not significant, $F(1,25)=2.16, p=.154$, $M S E=001, \eta_{p}^{2}=080$, nor were the two-way other interactions, $F \mathrm{~s}<1$. 


\section{Discussion}

In this experiment, participants respond faster when targets and flankers were compatible than when they are incompatible. Responses were slower when the arrows appeared within the same object rather than in different objects, also consistent with results of Experiments 1 and 2. Notably, as observed in Experiment 1, the flanker compatibility effect did not vary with whether the target and flankers appeared on the same object, and it was significant in the two conditions. The similarity of this result to that of Experiment 1, in which the three small objects were separated by two small gaps, and the difference from Experiment 2, in which they were not identical, implies that attention may extend from the object occupied by the target to the objects occupied by the flankers. This result is consistent with the prediction of the perceptual grouping account. The search prioritization view and integrality hypothesis also can explain the result.

\section{Experiments 4A and 4B}

As shown in Fig. 1, in Experiments 4A and 4B we used the same-object and different-object stimuli from Experiments 2 and 3, respectively, and the letter-identity flanker task of Richard et al.'s Experiments 4 and 5 and Shomstein \& Yantis (2002). To do so was to verify that the pattern of results in Experiments 2 and 3 could be obtained when the stimuli were not arrows.

The arrow-flanker task itself used in Experiments 1 to 3 likely involves processes of grouping that are stronger for congruent than incongruent trials, as in the congruent condition the arrows point to the same direction. This process of grouping may interact with the grouping and segmentation processes of the objects occupied by the arrows. Experiment 4 may help to rule out this possibility. In Experiments 1 to 3 and prior studies (e.g., Richard et al., 2008; Shomstein \& Yantis, 2002), whether the flanker compatibility effect was modulated by object-based attention was invariant of horizontal or vertical display orientation. Therefore, in the present experiment, we used only the horizontal display.

The predictions of the search prioritization view and integrality hypothesis are identical to those in Experiments 1 to 3 . Likewise, the predictions of the perceptual grouping account in Experiments $4 \mathrm{~A}$ and $4 \mathrm{~B}$ are identical to those in Experiments 2 and 3, respectively.

\section{Method}

Participants Fifty-two undergraduate students took part in the experiment for payment. Half (14 males and 12 females) were recruited for Experiment 4A and half (10 males and 16 females) for Experiment 4B.

Apparatus and stimuli The apparatus and stimuli were identical with Experiment 1, except that the target and flankers were replaced by four letters $H, V, U$ and $X$ (see Fig. 2), and the stimuli in the different-object condition in Experiments 4A and 4B were identical to those in Experiments 2 and 3, respectively. Each letter measured $0.7^{\circ} \times 0.7^{\circ}$.

Procedure and design The procedure was identical to that in Experiment 1 with the following exceptions. The target letter always appeared in the center of the display, with two flanking letters on either side. Moreover, the display only was horizontally arranged.

There were two trial blocks for each experiment, with a rest interval of $30 \mathrm{~s}$ between them. Each block consisted of 24 practice trials followed by 96 test trials. If the target was $H$ or $V$ and the flankers were two instances of $U$ or $X$, participants responded by pressing the $\mathrm{C}$ key, and if the target was $U$ or $X$ and the flankers were two instances of $H$ or $V$, participants responded by pressing the $\mathrm{M}$ key on the keyboard. In the compatible condition, the targets and flankers were always different letters from the same response category (e.g., $H$ target and $V$ flankers). Each experiment had a 2 (object condition: same, different) $\times 2$ (flanker compatibility: compatible, incompatible) design.

\section{Results: Experiment 4A}

Mean RTs for the correct trials and PEs were calculated for each participant as a function of object condition (same, different), flanker compatibility (compatible, incompatible; see Tables 1 and 2). They then were submitted to separate ANOVAs.

Reaction time Responses were faster in the different-object condition $(550 \mathrm{~ms})$ than in the same-object condition (562 $\mathrm{ms}), F(1,25)=13.31, p<.001, M S E=215, \eta_{p}{ }^{2}=.409$, and in the compatible condition $(548 \mathrm{~ms})$ than in the incompatible condition (563 ms), $F(1,25)=19.05, p<.001, M S E=284$, $\eta_{p}{ }^{2}=.433$. Object condition interacted with flanker compatibility, $F(1,25)=6.54, p=.017, M S E=252, \eta_{p}{ }^{2}=.207$, with the flanker compatibility effect being larger in the same-object condition $(22 \mathrm{~ms})$ than in the different-object condition $(7 \mathrm{~ms}$; see Fig. 3), $t(25)=2.56, p=.017$. Further analysis showed that the flanker compatibility effect was significant in the sameobject condition, $t(25)=4.82, p<.001$, but not in the different-object condition, $t(25)=1.57, p=.129$.

Percent error The main effect of object condition was not significant, $F(1,25)=1.44, p=.242, M S E=.001, \eta_{p}^{2}=.054$, 
nor were the main effect of flanker compatibility and the interaction between object condition and flanker compatibility $\left(F_{\mathrm{S}}<1\right)$.

\section{Results: Experiment 4B}

The data were analyzed as for Experiment 4A.

Reaction time Responses were faster in the compatible condition $(552 \mathrm{~ms})$ than in the incompatible condition $(574 \mathrm{~ms})$, $F(1,25)=14.33, p<.001, M S E=843, \eta_{p}{ }^{2}=.364$. Responses tended to be faster in the different-object condition $(560 \mathrm{~ms})$ than in the same-object condition $(567 \mathrm{~ms})$, but it was not significant, $F(1,25)=2.24, p=.147, M S E=573, \eta_{p}{ }^{2}=$ .082. Object condition did not interact with flanker compatibility $(F<1)$. Additional analysis showed that the flanker compatibility effects were reliable in the same-object ( 24 $\mathrm{ms})$ and different-object $(19 \mathrm{~ms})$ conditions, $t(25)=3.32, p$ $=.003 ; t(25)=2.81, p=.009$. Furthermore, comparison of the RT results in the different-object condition for Experiments 4A and 4B showed the flanker compatibility effect was smaller when the small objects were identical (Experiment 4A) than when they were different (Experiment 4B), $t(25)=$ $2.10, p=.046$.

Percent error The main effects of object condition and flanker compatibility were not significant $\left(F_{\mathbf{S}}<1\right)$, nor was their interaction, $F(1,25)=2.63, p=.118, M S E=.001, \eta_{p}{ }^{2}=.095$.

\section{Discussion}

In this experiment, participants respond faster when targets and flankers were compatible than when they are incompatible. Responses were or tended to be slower when the letters appeared within the same object rather than in different objects, also consistent with results of Experiments 1 to 3 .

Importantly, in Experiment 4A, a significantly larger flanker compatibility effect was observed when flankers and target appeared in the same object than when they appeared in the different objects, whereas that was not the case in Experiment 4B. Moreover, the flanker compatibility effect in the differentobject condition was smaller when the small objects were different in Experiment 4A than when they were identical in Experiment 4B. This result is consistent with the prediction of the perceptual grouping account, whereas the search prioritization view and integrality hypothesis cannot explain the modulation of flanker compatibility effect by object condition in Experiment 4A.

This result also rules out a possibility that the arrow-flanker task itself used in Experiments 1 to 3 involves processes of grouping that are stronger for congruent than incongruent trials, which could interact with the grouping and segmentation processes of the irrelevant background objects.

\section{General discussion}

In the current experiments, we distinguished the search prioritization, integrality, and perceptual grouping accounts of object-based intentional spreading. We did so by investigating whether, when the target and flankers are displayed in the same or different objects, perceptual grouping of these background objects occupied by target and flankers can affect the target-flanker interference.

In Experiments 1, 3, and 4B, when the three small objects occupied by target and flankers were completely identical or separated by two small gaps and the same object condition was similar to that of the prior studies, the target-flanker interference effect was not smaller when the target and flankers were in different objects than in the same object. This result implies that attention likely extends across different objects or, in other words, that gaps may not impede attentional extending across the different objects occupied by the target and flankers. These results are consistent with those observed in Shomstein \& Yantis (2002, Experiments 1 to 4) and Richard et al. (2008, Experiments 4 and 5). Moreover, these results are similar to those of prior studies using other techniques, which have demonstrated that attention can extend from target to flankers that share a basic feature such as closure, color, contour, and movement (e.g., Driver \& Baylis, 1989; Fuentes et al., 1998; Harms \& Bundesen, 1983; Kim \& Cave, 2001; Kramer \& Jacobson, 1991). However, different from those prior findings obtained when target and flankers share a basic feature, the results of Experiments 1, 3, and 4B further suggest that attention likely extends across objects occupied by target and flankers when these objects share a similar or identical shape.

The central finding of the current study is that there was a larger flanker compatibility effect in the same-object condition than in the different-object condition in Experiments 2 and 4A, in which, for the different-object condition, the target and flankers were displayed in objects that differed in shape. Moreover, the flanker compatibility effect in the differentobject condition was smaller when the small objects were different in Experiment 4A than when they were identical in Experiment 4B. This finding indicates that attentional spreading was greater within a single object than across different objects, which is likely due to attentional spreading being impeded when these objects occupied by the target and flankers were different in shape. These results cannot be explained well by the integrality hypothesis, given that the taskrelevant features were not integral to the objects in a display and did not contribute to the shape or surface structure of the objects. Also, these results cannot be explained well by the 
search prioritization view, as the target location was fixed. In addition, the results of Experiments 2 and $4 \mathrm{~A}$ can be explained by the attention shift hypothesis, which states that object structure may influence shifts of attention, with a shift in the spatial locus of attention either more efficient or more likely within an object than between objects (Egly et al., 1994; Lamy \& Egeth, 2002). However, the attention shift hypothesis cannot easily explain the results of Experiments 1, 3, and 4B, in which there was not a larger flanker compatibility effect in the same-object condition than in the different-object condition.

The findings of Experiments 1 to 4 together suggest that when the target and flankers are displayed in the same or different objects, perceptual grouping (created by shape similarity) of these background objects occupied by target and flankers in the different object condition, can affect the target-flanker interference. This finding can explain the disparities in the results of previous studies. In Shomstein \& Yantis (2002, 2004), Zhao et al. (2013), and Experiments 4 and 5 of Richard et al. (2008), for the different-object condition, the three objects were identical or similar (see Fig. 1). Consequently, object-based attention could spread across the objects, resulting in the target-flanker interference effect being invariant of whether the target and flankers were displayed in a single object or different objects. By contrast, in Experiments 1 to 3 of Richard et al. (2008), in the incompatible and different-object condition, the central object was different from the two flanking objects, which might impede the attentional spreading across the three different objects, resulting in a smaller target-flanker interference effect in the different-object than same-object condition.

In the current Experiments 1 to 4, and previous studies (e.g., Chen \& Cave, 2006, 2008; Ho, 2011; Experiments 4 and 5 of Richard et al., 2008), the object-based attention effect was negative; that is, the target-identification response was slower in the same object condition than in the different-object condition. The negative object-based effects may due to an extra segmentation process that is required to separate irrelevant information (i.e., flankers) from the relevant information (i.e., the target) when they appear on the same object; this segmentation stage may not be required in the different object condition, because the target and flankers appear on three different objects, which are already perceptually segmented from each other. Moreover, this negative object-based attention effect is also likely to arise from the target not being as discriminable in the same-object condition as in the different-object condition. However, whether this confusability has an effect on the interaction between object condition and the flanker compatibility effect needs to be investigated.

The current study differs from previous studies (e.g., Chen \& Cave, 2006, 2008; Ho, 2011) that also explored why a larger flanker compatibility effect was not observed in the same-object condition in Shomstein \& Yantis (2002) and Experiments 4 and 5 of Richard et al. (2008). For example,
Chen \& Cave (2006) argued that subjective parsing of the display influences the occurrence of object-based attention when the target position is certain. They employed a task and stimuli similar to those of Shomstein \& Yantis (2002) and induced participants to see the whole stimulus pattern as two separate objects. However, unlike Shomstein \& Yantis (2002), they reported larger flanker compatibility effects when the target and the flankers were within the same object.

Another study by Chen \& Cave (2008) presented two targets on two ends of the same object, or on different objects, and asked participants to make same/different responses to the targets. Before onset of the targets, an endogenous cue appeared in the center of the display to indicate the target locations with $100 \%$ validity. Performance was found to be better when the two targets were on the same object rather than on different objects. Ho (2011) argued that the RT-based measure is less sensitive than a data-limited accuracy measure in reflecting the quality of perceptual representations, so it is not sufficiently a strong evidence to distinguish between sensory enhancement and search prioritization accounts. Using a flanker task similar to that of Shomstein \& Yantis (2002) and adopting the data-limited accuracy measure, Ho reported that attention could spread within the attended object.

In the current study, although the RT-based measure was used and top-down cues were not given to participants, we found a larger flanker compatibility effect in the same-object condition than in the different-object condition, suggesting that these factors were not the direct determinants. The larger flanker compatibility effect observed in those studies (e.g., Chen \& Cave, 2006, 2008; Ho, 2011) may be because their manipulation affected attentional spreading. That is, top-down cues and the datalimited accuracy measure likely caused the object occupied by the target to be segmented from the objects occupied by flankers, which could impede or attenuate attentional spreading across objects, resulting in the reduction of the flanker compatibility effect in the different-object condition.

In addition, our findings also can explain the result observed in Experiment 5 of Shomstein \& Yantis (2002). In that experiment, a small, bright square was flashed briefly in the center of the central rectangle (see Fig. 1, middle panel), and it was uninformative about the location of the upcoming target but was presumed to summon attention to the central rectangle. After a 100-ms interstimulus interval (ISI), the target and three nontargets appeared. The target appeared either in the cued rectangle or in one of the uncued rectangles. The two flankers never appeared in the same rectangle as the target (a third, nonsense symbol was presented to balance the display and prevent apparent motion effects). Shomstein \& Yantis (2002) reported a smaller target-flankers interference effect when the target appeared in the cued object (that is, the large object) than when it appeared in the uncued object (that is, one small object). These authors argued that when attention could not be narrowly focused in advance, object-based modulation 
of the flanker effect was observed. Our explanation was that the cue displayed in the large object may have it be segmented from the small objects, which may impede or attenuate attentional spreading across these objects, resulting in a small target-flankers interference effect when the target appeared in the large object and the flankers appeared in the small objects. However, when the target appeared in one of the small objects and the flankers appeared in the cued large object, which may enhance the processing of flankers, resulting in a large target-flankers interference effect.

In conclusion, even when the task-relevant features do not contribute to the shape or surface structure of the objects, attention may spread from the object occupied by the target to the different objects occupied by the flankers when these objects have similar or identical shape. Such attentional spreading is impeded when the objects have different shape, suggesting that object-based attentional spreading is modulated by the perceptual structure of objects.

Acknowledgments This research was supported in part by grants from the National Science Foundation of China (31470984). We wish to thank Juan Lupiáñez and Shaun P. Vecera for their helpful comments on earlier versions of the manuscript.

\section{References}

Baldauf, D., \& Desimone, R. (2014). Neural mechanisms of object-based attention. Science, 344, 424-427.

Baylis, G. C., \& Driver, J. (1992). Visual parsing and response competition: The effect of grouping factors. Perception \& Psychophysics, $51,145-162$.

Behrmann, M., Zemel, R. S., \& Mozer, M. C. (1998). Object-based attention and occlusion: Evidence from normal participants and a computational model. Journal of Experimental Psychology: Human Perception and Performance, 24, 1011-1036.

Brown, J. M., \& Denney, H. I. (2007). Shifting attention into and out of objects: Evaluating the processes underlying the object advantage. Perception \& Psychophysics, 69, 606-618.

Chen, Z. (2012). Object-based attention: A tutorial review. Attention, Perception, \& Psychophysics, 74, 784-802.

Chen, Z., \& Cave, K. (2006). Reinstating object-based attention under positional certainty: The importance of subjective parsing. Perception \& Psychophysics, 68, 992-1003.

Chen, Z., \& Cave, K. (2008). Object-based attention with endogenous cuing and positional certainty. Perception \& Psychophysics, 70, $1435-1443$.

Cho, Y. S., \& Proctor, R. W. (2003). Stimulus and response representations underlying orthogonal stimulus-response compatibility effects. Psychonomic Bulletin \& Review, 10, 45-73.

Cosman, J. D., \& Vecera, S. P. (2012). Object-based attention overrides perceptual load to modulate visual distraction. Journal of Experimental Psychology: Human Perception and Performance, $38,576-579$.

Cousineau, D. (2007). Confidence intervals in within-subject designs: A simpler solution to Loftus and Masson's method. Tutorials in Quantitative Methods for Psychology, 1, 42-45.

Desimone, R., \& Duncan, J. (1995). Neural mechanisms of selective visual attention. Annual Review of Neuroscience, 18, 193-222.
Driver, J., \& Baylis, G. C. (1989). Movement and visual attention: The spotlight metaphor breaks down. Journal of Experimental Psychology: Human Perception and Performance, 15, 448-456.

Duncan, J. (1984). Selective attention and the organization of visual information. Journal of Experimental Psychology: General, 113, 501-517.

Egly, R., Driver, J., \& Rafal, R. D. (1994). Shifting visual attention between objects and locations: Evidence from normal and parietal lesion subjects. Journal of Experimental Psychology: General, 123, 161-177.

Eriksen, B. A., \& Eriksen, C. W. (1974). Effects of noise letters upon the identification of a target letter in a nonsearch task. Perception \& Psychophysics, 16, 143-149.

Eriksen, C. W., \& Hoffman, J. E. (1973). The extent of processing of noise elements during selective encoding from visual displays. Perception \& Psychophysics, 14, 155-160.

Fuentes, L. J., Humphreys, G. W., Agis, I. F., Carmona, E., \& Catena, A. (1998). Object-based perceptual grouping affects negative priming. Journal of Experimental Psychology: Human Perception and Performance, 24, 664-672.

Goldsmith, M., \& Yeari, M. (2003). Modulation of object-based attention by spatial focus under endogenous and exogenous orienting. Journal of Experimental Psychology. Human Perception and Performance, 29, 897-918.

Harms, L., \& Bundesen, C. (1983). Color segregation and selective attention in a nonsearch task. Perception \& Psychophysics, 33, 11-19.

Ho, M. C. (2011). Object-based attention: Sensory enhancement or scanning prioritization? Acta Psychologica, 138, 45-51.

Hollingworth, A., Maxcey-Richard, A. M., \& Vecera, S. P. (2012). The spatial distribution of attention within and across objects. Journal of Experimental Psychology: Human Perception and Performance, $38,135-151$

Kim, M.-S., \& Cave, K. R. (2001). Perceptual grouping via spatial selection in a focused-attention task. Vision Research, 41, 611-624.

Kramer, A. F., \& Jacobson, A. (1991). Perceptual organization and focused attention: The role of objects and proximity in visual processing. Perception \& Psychophysics, 50, 267-284.

Lamy, D., \& Egeth, H. (2002). Object-based selection: The role of attentional shifts. Perception \& Psychophysics, 64, 52-66.

Lamy, D., Leber, A. B., \& Egeth, H. E. (2013). Selective attention. In I. B. Weiner, A. F. Healy, \& R. W. Proctor (Eds.), Experimental psychology (2nd ed., Vol. 4, pp. 267-294). Hoboken: Wiley.

Lamy, D., \& Tsal, Y. (2000). Object features, object locations and object files: Which does selective attention activate and when? Journal of Experimental Psychology: Human Perception \& Performance, 26, 1387-1400.

Luo, C., Lupiáñez, J., Funes, M. J., \& Fu, X. (2010). Modulation of spatial Stroop by object-based attention but not by space-based attention. The Quarterly Journal of Experimental Psychology, 63, 516-530.

Luo, C., Lupiáñez, J., Funes, M. J., \& Fu, X. (2011). The modulation of spatial congruency by object-based attention: Analysing the "locus" of the modulation. The Quarterly Journal of Experimental Psychology, 64, 2455-2469.

Posner, M. I. (1980). Orienting of attention. Quarterly Journal of Experimental Psychology, 32, 3-25.

Reppa, I., Schmidt, W. C., \& Leek, E. C. (2012). Successes and failures in producing attentional object-based cueing effects. Attention, Perception, \& Psychophysics, 74, 43-69.

Richard, A. M., Lee, H., \& Vecera, S. P. (2008). Attentional spreading in object-based attention. Journal of Experimental Psychology: Human Perception and Performance, 34, 842-853.

Scholl, B. J. (2001). Objects and attention: The state of the art. Cognition, $80,1-46$. 
Shomstein, S., \& Behrmann, M. (2008). Object-based attention: Strength of object representation and attentional guidance. Perception \& Psychophysics, 70, 132-144.

Shomstein, S., \& Yantis, S. (2002). Object-based attention: Sensory modulation or priority setting? Perception \& Psychophysics, 64, 41-51.

Shomstein, S., \& Yantis, S. (2004). Configural and contextual prioritization in object-based attention. Psychonomic Bulletin \& Review, 11, $247-253$.
Vecera, S. P. (1994). Grouped locations and object-based attention: Comment on Egly, Driver, and Rafal (1994). Journal of Experimental Psychology: General, 123, 316-320.

Weinbach, N., \& Henik, A. (2012). The relationship between alertness and executive control. Journal of Experimental Psychology: Human Perception and Performance, 38, 1530-1540.

Zhao, J., Kong, F., \& Wang, Y. (2013). Attentional spreading in objectbased attention: The roles of target-object and target presentation time. Attention, Perception, \& Psychophysics, 75, 876-887. 